\title{
The Two-hop MISO Broadcast Network with Quantized Delayed CSIT
}

\author{
Zhao Wang, Chao Wang, Ming Xiao, and Mikael Skoglund \\ Communication Theory Lab, Royal Institute of Technology (KTH), Stockholm, Sweden \\ Email: \{zhaowang, chaowang, mingx, skoglund\}@kth.se
}

\begin{abstract}
We consider a downlink two-hop MISO broadcast network with a 2 -antenna source communicating to 2 singleantenna destinations, assisted by 2 single-antenna intermediate relays. We investigate spectrally efficient transmission schemes and their achieved sum degrees of freedom (DoF), with quantized delayed channel state information (CSI) feedback. Assuming Grassmannian vector quantization, we study two feedback scenarios according to the feedback range limit, namely global-range feedback, i.e., the source can receive the feedback signals from both the relays and the destinations, and one-hop-range feedback, i.e., each node can only attain the feedback information of its upcoming hop. We establish a sum DoF lower bound for each case. Our results reveal that when the quantization rate at relays $B_{R}=\alpha_{1} \log _{2}(S N R)$ and at destinations $B_{D}=\alpha_{2} \log _{2}(S N R)$ for $\min \left\{\alpha_{1}, \alpha_{2}\right\} \geq 1$, the optimal sum DoF $\frac{4}{3}$ can be achieved with finite-rate delayed feedback.
\end{abstract}

\section{INTRODUCTION}

Considering a downlink broadcast channel with a multipleantenna transmitter and single-antenna receivers, the channel state information at transmitter (CSIT) plays a crucial role of providing degrees of freedom (DoF) gain. In order to obtain CSIT, the transmitter generally requires the estimated CSI feedback from receivers. A feasible strategy in practice is to deploy a finite-rate feedback scheme, in which each receiver feeds back a finite number of bits regarding its input channel realization. Reference [1] showed that if each receiver sends back quantized CSI, the full DoF gain can still be achieved when the number of quantization bits scales linearly with $\log _{2}(S N R)$, in the high signal-to-noise ratio (SNR) region. However, feedback delay will bring notable DoF loss if it approaches or exceeds channel coherence time [2]. Recently, a pioneer work by Maddah-Ali and Tse in [3] considered this delay issue in the downlink MISO channel. They proved that when the feedback delay exceeds the channel coherence time such that the CSIT is completely outdated, via a careful transmission and interference alignment (IA) design (we term it as MAT scheme), a promising DoF gain can still be realized. However, a potential problem of this model is that capacityunlimited feedback channels are assumed to guarantee the delayed CSIT to perfect. Based on practical concerns, finiterate feedback with random vector quantization is used in reference [4] to show that the DoF performance of the MAT scheme maintains, when the number of feedback bits scales linearly with $\log _{2}(S N R)$.

Nowadays, due to the constantly increasing demands of higher spectral efficiency, energy efficiency, and device mobil- ity, deploying relays to assist in wireless communication has started to draw substantial research attention. In our previous work [5], we considered downlink MISO networks in which the signals from a multi-antenna source cannot directly reach its multiple single-antenna destinations so that intermediate relays are used to aid communication. DoF gains in these multi-hop networks were exhibited, with perfect delayed CSIT delivered by infinite-rate feedback signals from the relays and destinations. In this paper, from a more practical perspective, we step further to consider finite-rate delayed CSI feedback. Specifically, a 2-user 2-hop downlink MISO network is studied. The feedback rates of relays and destinations are limited by $B_{R}$ and $B_{D}$ bits, respectively. We apply the Grassmannian vector quantization [6] [7] at each receiver and propose two transmission schemes along with two different feedback scenarios. In a global-range feedback scenario, where the quantized delayed CSI regarding both hops can be delivered to the source, an equivalent MAT scheme is employed. On the other hand, when feedback occurs within only one hop in the one-hop-range feedback scenario, an IA-based scheme is proposed. We establish an achievable sum DoF lower bound for each scheme, with respect to the quantization errors at the relays and destinations. Our results explicitly show that when the quantization rates scale linearly with $\log _{2}(S N R)$, i.e., $B_{R}=\alpha_{1} \log _{2}(S N R)$ and $B_{D}=\alpha_{2} \log _{2}(S N R)$, the optimal sum DoF $\frac{4}{3}$ can be achieved in both feedback scenarios, if $\min \left\{\alpha_{1}, \alpha_{2}\right\} \geq 1$. Therefore, finite-rate delayed feedback is still advantageous, in terms of DoF performance.

Notations: The superscript ${ }^{T}$, and ${ }^{*}$ represent the transpose and Hermitian transpose operation, respectively. $|\mathbf{a}|$ and $|a|$ represent the Euclidean norm of the vector a and absolute value of the scalar $a$, respectively. $|\mathbf{A}|$ denotes the determinant of the matrix $\mathbf{A}$. $\mathbf{A} \equiv\left[a_{i j}\right]$ denotes that the matrix $\mathbf{A}$ is constituted in such a way that the element on its $i$ th row and $j$ th column is $a_{i j} . \mathbb{E}(\cdot)$ represents an expectation operation. $(\alpha)^{+}$denotes $\max \{0, \alpha\}$.

\section{SySTEM MODEL}

As shown in Fig. 1, we consider a 2-user 2-hop MISO broadcast network in which a source node $S$ with 2 transmit antennas intends to send independent messages to 2 singleantenna destinations $D_{1}$ and $D_{2}$. There is no physical link between them and 2 single-antenna relays $R_{1}$ and $R_{2}$ are deployed to carry out the communication. 


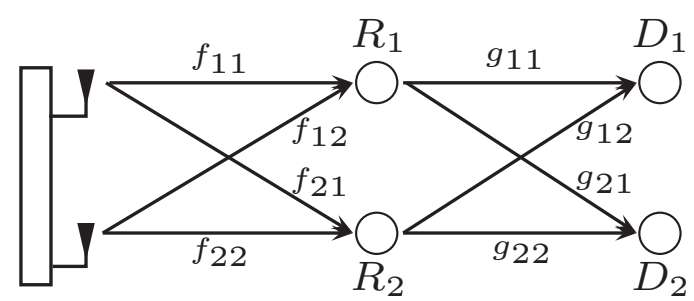

Fig. 1. A 2-user 2-hop MISO broadcast network.

Let $\mathcal{C}_{\Sigma}(P)$ denote the sum capacity of the considered network with transmit power $P$. The sum DoF is defined as

$$
d=\lim _{P \rightarrow \infty} \frac{\mathcal{C}_{\Sigma}(P)}{\log _{2} P} .
$$

Assume a block fading environment, where the source-relay channel gains $\left\{f_{i j}(t)\right\}$ and the relay-destination channel gains $\left\{g_{i j}(t)\right\}(i, j \in\{1,2\})$ remain constant within one time slot but change independently across different time slots following independent and identically distributed (i.i.d.) Rayleigh distributions. We denote the source's transmission signal at time slot $t$ as $\mathbf{x}(t)=\left[x_{1}(t) x_{2}(t)\right]^{T}$, where $x_{i}(t)(i \in\{1,2\})$ is the transmission signal of antenna $i$. The received signal of relay $R_{i}(i \in\{1,2\})$ at time slot $t$ is

$$
y_{R_{i}}(t)=f_{i 1}(t) x_{1}(t)+f_{i 2}(t) x_{2}(t)+z_{i}(t),
$$

where $z_{i}(t)$ is the i.i.d. zero-mean unit-variance complex noise. At time slot $t, R_{i}$ transmits $x_{R_{i}}(t)$ so that in the second hop, the received signal of destination $D_{k}(k \in\{1,2\})$ is

$$
y_{D_{k}}(t)=g_{k 1}(t) x_{R_{1}}(t)+g_{k 2}(t) x_{R_{2}}(t)+n_{k}(t),
$$

where $n_{k}(t)$ is the i.i.d. zero-mean unit-variance complex noise. We consider full-duplex relaying and for presentation simplicity the processing delay at relays is omitted. We assume an equal power allocation at the source and the average transmission power is limited by $P$. In addition, the average transmission power at each relay is limited by $\frac{P}{2}$.

Quantized delayed CSIT feedback is considered in this paper. At each time slot $t$, each relay $R_{i}(i \in\{1,2\})$ can obtain the perfect instantaneous input channel vector $\mathbf{f}_{i}(t)=$ $\left[f_{i 1}(t) f_{i 2}(t)\right]^{T}$ via training. It can pass its channel knowledge to the destinations along with actual data transmission. In addition, $R_{i}$ quantizes $\mathbf{f}_{i}(t)$ into $\hat{\mathbf{f}}_{i}(t)=\left[\hat{f}_{i 1}(t) \hat{f}_{i 2}(t)\right]^{T}$ using a $B_{R}$-bit quantizer and then broadcasts the $B_{R}$-bit quantized CSI back to the sources. Similarly, each destination $D_{i}$ is assumed to know $\mathbf{g}_{i}(t)=\left[g_{i 1}(t) g_{i 2}(t)\right]^{T}$ perfectly by training. $\mathbf{g}_{i}(t)$ is quantized into $\hat{\mathbf{g}}_{i}(t)=\left[\hat{g}_{i 1}(t) \hat{g}_{i 2}(t)\right]^{T}$ by a $B_{D}$-bit quantizer for broadcasting. We assume the feedback delay is longer than the channel coherence time. Thus the CSIT is considered as completely outdated to the current time instant and the feedback delay is one time slot. Two feedback scenarios are considered and defined as follows:

1) Global-range quantized delayed feedback: The source $S$ can successfully decode the feedback signals from both relays and destinations. Henceforth, $S$ knows $\hat{\mathbf{f}}_{i}(t)$ and $\hat{\mathbf{g}}_{i}(t)$ at time slot $t+1$.
2) One-hop-range quantized delayed feedback: In this scenario, the feedback only occurs within one hop. Thus $S$ can only attain $\hat{\mathbf{f}}_{i}(t)$ and $R_{j}$ can obtain $\hat{\mathbf{g}}_{i}(t)$, at time slot $t+1$.

\section{BACKGROUND}

In this section, we first briefly introduce some preliminary results on the Grassmannian vector quantization scheme [6] [7] and then state the sum DoF of the 2-user 2-hop MISO broadcast network with perfect delayed CSIT.

\section{A. Quantization scheme}

Let $B$ denote quantization rate. The vector quantization scheme works as follows: for an arbitrary $m \times 1$ unit-norm complex vector $\mathbf{w}$, the quantizer tries to map it into an $m \times 1$ unit-norm vector $\hat{\mathbf{w}}$, chosen from a quantization codebook $\mathcal{A}=\left\{\hat{\mathbf{w}}_{1}, \hat{\mathbf{w}}_{2}, \ldots, \hat{\mathbf{w}}_{2^{B}}\right\}$. The quantization criterion is

$$
\hat{\mathbf{w}}=\arg \max _{\hat{\mathbf{w}} \in \mathcal{A}}\left\{\left|\hat{\mathbf{w}}^{*} \mathbf{w}\right|\right\} .
$$

Similar to [6] [7], the quantization codebook is chosen as the solution of Grassmannian line-packing problem [8].

Quantization error: The quantization error based on the codebook $\mathcal{A}$ can be defined as the minimum distance of any pair of lines represented by the codewords, which is the sine of the smallest angle between them:

$$
\epsilon(\mathcal{A})=\min _{1 \leq k<l \leq 2^{B}} \sqrt{1-\left|\hat{\mathbf{w}}_{k}^{*} \hat{\mathbf{w}}_{l}\right|^{2}}=\sin \left(\theta_{\min }\right) .
$$

The Grassmannion quantization error can be bounded as [9]:

$$
2^{-\frac{B}{2(m-1)}} \leq \sin \left(\theta_{\min }\right) \leq 2\left(2^{-\frac{B}{2(m-1)}}\right) .
$$

Channel state quantization: The quantization codebook applied at relays is $\mathcal{A}_{R}=\left\{\hat{\mathbf{r}}_{1}, \hat{\mathbf{r}}_{2}, \ldots, \hat{\mathbf{r}}_{2^{B} R}\right\}$. That applied at destinations is $\mathcal{A}_{\mathcal{D}}=\left\{\hat{\mathbf{d}}_{1}, \hat{\mathbf{d}}_{2}, \ldots, \hat{\mathbf{d}}_{2^{B_{D}}}\right\}$. The codebooks are assumed to be known by all terminals. Note that the Grassmannian vector quantization applies to unit-norm vector space. Thus only the directions of the channel state vectors $\mathbf{f}_{i}(t)$ and $\mathbf{g}_{i}(t)$ are quantized. Similar to (5), we define the quantization errors at relays and destinations as $\sin \left(\theta_{f}\right)$ and $\sin \left(\theta_{g}\right)$, respectively. They can be bounded using the quantization rates $B_{R}$ and $B_{D}$ as (6).

\section{B. With perfect delayed CSIT}

If perfect delayed CSIT with infinite feedback rate can be attained, we have the following result regarding the sum DoF of the considered network [5].

Theorem 1: The sum DoF of the 2-user 2-hop MISO broadcast network with either perfect global-range delayed feedback or perfect one-hop-range delayed feedback is $\frac{4}{3}$.

Theorem 1 implies that the optimal sum DoF $\frac{4}{3}$ can be achieved if there is no error in the delayed CSIT. In the following sections, we will introduce transmission schemes based on quantized delayed feedback and discuss the sum DoF loss in different scenarios.

\section{Global-RANGE QUANTIZED DELAYED FEEDbACK}

In this section, we consider transmissions regarding the global-range quantized delayed feedback. 


\section{A. Transmission scheme}

The transmission scheme follows that proposed in [3] but based on an equivalent MISO broadcast channel with quantized delayed CSI feedback. Hence, we term it as an equivalent MAT scheme. Note that we consider the high SNR regime so that the unit-power Gaussian noise is omitted for presentation simplicity. The transmission process contains two phases and takes 3 time slots.

Phase one: Individual message broadcast. This phase takes 2 time slots. Let $\mu_{i}$ and $\nu_{i}$ represent two independent messages (encoded using Gaussian random codes) intended for destination $D_{i}, i \in\{1,2\}$. At the first time slot, the source uses its two antennas to broadcast $\mu_{1}$ and $\nu_{1}$ respectively, i.e. $\mathbf{x}(1)=\left[\begin{array}{ll}\mu_{1} & \nu_{1}\end{array}\right]^{T}$. At the second time slot, it broadcasts $\mu_{2}$ and $\nu_{2}$ so that $\mathbf{x}(2)=\left[\begin{array}{ll}\mu_{2} & \nu_{2}\end{array}\right]^{T}$. According to (2), we have

$$
y_{R_{i}}(t)=\left[f_{i 1}(t) f_{i 2}(t)\right]\left[\begin{array}{ll}
\mu_{t} & \nu_{t}
\end{array}\right]^{T} \text {. }
$$

We consider amplify-and-forward relaying and define $\Lambda(t)=$ $\operatorname{diag}\left\{\lambda_{1}(t), \lambda_{2}(t)\right\}$, where $\lambda_{i}(t)$ represents the amplification coefficient of relay $R_{i}$ at time slot $t . \lambda_{i}(t)$ is chosen such that $\lambda_{i}^{2}(t)\left(f_{i 1}^{2}(t)+f_{i 2}^{2}(t)\right)=1$. Hence, we have $\lambda_{i}(t)=\frac{1}{\left|\mathbf{f}_{i}(t)\right|}$. Now substituting $x_{R_{i}}(t)=\lambda_{i}(t) y_{R_{i}}(t)$ into (3), the received signals at destinations are as follows:

$$
y_{D_{i}}(t)=\left[g_{i 1}(t) g_{i 2}(t)\right] \Lambda(t)\left[\begin{array}{ll}
f_{11}(t) & f_{12}(t) \\
f_{21}(t) & f_{22}(t)
\end{array}\right]\left[\begin{array}{c}
\mu_{t} \\
\nu_{t}
\end{array}\right] .
$$

Let $\overline{\mathbf{f}}_{i}(t)^{T}=\left[\bar{f}_{i 1}(t) \bar{f}_{i 2}(t)\right]=\frac{1}{\mid \mathbf{f}_{i}(t)[}\left[f_{i 1}(t) f_{i 2}(t)\right]$ and $\overline{\mathbf{g}}_{i}(t)^{T}=\left[\bar{g}_{i 1}(t) \bar{g}_{i 2}(t)\right]=\frac{1}{\left|\mathbf{g}_{i}(t)\right|}\left[g_{i 1}(t) g_{i 2}(t)\right]$. Define $\bar{y}_{D_{i}}(t)=y_{D_{i}}(t) /\left|\mathbf{g}_{i}(t)\right|$ as the normalized received signal at destination $D_{i}$. We have:

$$
\begin{aligned}
\bar{y}_{D_{i}}(t)= & \left(\bar{g}_{i 1}(t) \bar{f}_{11}(t)+\bar{g}_{i 2}(t) \bar{f}_{21}(t)\right) \mu_{t} \\
& +\left(\bar{g}_{i 1}(t) \bar{f}_{12}(t)+\bar{g}_{i 2}(t) \bar{f}_{22}(t)\right) \nu_{t} .
\end{aligned}
$$

Meanwhile, at each time slot, the relays and destinations quantize their input channel vectors and feed the quantization indexes to the source. Thus, after the first phase, the quantized channel vectors $\hat{\mathbf{f}}_{i}(1), \hat{\mathbf{f}}_{i}(2), \hat{\mathbf{g}}_{i}(1), \hat{\mathbf{g}}_{i}(2), \forall i \in\{1,2\}$ are known at $S$.

Phase two: Common equation delivery. Phase two occurs at the third time slot. Because $S$ has the quantized CSI regarding the first phase, combining the messages $\mu_{1}, \mu_{2}, \nu_{1}$, and $\nu_{2}$, it can transmit the following signal:

$$
\mathbf{x}(3)=\left[\hat{y}_{D_{2}}(1)+\hat{y}_{D_{1}}(2) 0\right]^{T},
$$

where $\hat{y}_{D_{i}}(t)$ represents the signal in (9) with the channel vectors replaced with their quantized version. Let $R_{1}$ amplify and forward its received signal, using amplification coefficient $\lambda_{1}(3)=\frac{1}{f_{11}(3)}$, and $R_{2}$ remains silent. The received signal at destination $D_{i}$ is:

$$
\begin{aligned}
y_{D_{i}}(3) & =g_{i 1}(3) \lambda_{1}(3) f_{11}(3)\left(\hat{y}_{D_{2}}(1)+\hat{y}_{D_{1}}(2)\right) \\
& =g_{i 1}(3)\left(\hat{y}_{D_{2}}(1)+\hat{y}_{D_{1}}(2)\right) .
\end{aligned}
$$

Define matrices $\overline{\mathbf{F}}(t) \equiv\left[\bar{f}_{i j}(t)\right], \overline{\mathbf{G}}(t) \equiv\left[\bar{g}_{i j}(t)\right], \hat{\mathbf{F}}(t) \equiv$ $\left[\hat{f}_{i j}(t)\right]$ and $\hat{\mathbf{G}}(t) \equiv\left[\hat{g}_{i j}(t)\right](i, j \in\{1,2\})$. Now we can write the received signals of $D_{1}$ during the two phases into a $3 \times 1$ vector $\mathbf{y}_{D_{1}}=\left[\bar{y}_{D_{1}}(1) \bar{y}_{D_{1}}(2) \frac{y_{D_{1}}(3)}{g_{11}(3)}\right]^{T}$. Equivalently, we have

$$
\mathbf{y}_{D_{1}}=\underbrace{\left[\begin{array}{cc}
\bar{h}_{11}(1) & \bar{h}_{12}(1) \\
0 & 0 \\
\hat{h}_{21}(1) & \hat{h}_{22}(1)
\end{array}\right]}_{\mathbf{H}_{D_{1}}}\left[\begin{array}{l}
\mu_{1} \\
\nu_{1}
\end{array}\right]+\underbrace{\left[\begin{array}{cc}
0 & 0 \\
\bar{h}_{11}(2) & \bar{h}_{12}(2) \\
\hat{h}_{11}(2) & \hat{h}_{12}(2)
\end{array}\right]}_{\mathbf{I}_{D_{2}}}\left[\begin{array}{c}
\mu_{2} \\
\nu_{2}
\end{array}\right],
$$

where $\bar{h}_{i j}(t)$ and $\hat{h}_{i j}(t)$ denote the elements on the $i$ th row and $j$ th column of matrices $\overline{\mathbf{H}}(t)=\overline{\mathbf{G}}(t) \overline{\mathbf{F}}(t)$ and $\hat{\mathbf{H}}(t)=$ $\hat{\mathbf{G}}(t) \hat{\mathbf{F}}(t)$, respectively. We observe that if no quantization error exists such that $\hat{h}_{i j}(t)=\bar{h}_{i j}(t)$, we have $\operatorname{rank}\left(\mathbf{H}_{D_{1}}\right)=2$ and $\operatorname{rank}\left(\mathbf{I}_{D_{2}}\right)=1$ almost surely. In addition, the signal space spanned by $\mathbf{H}_{D_{1}}$ has no overlapping with the interference space spanned by $\mathbf{I}_{D_{2}}$. By zero-forcing the interference, the DoF $\frac{2}{3}$ can be achieved. The similar analysis can be made on the received signals at $D_{2}$. Thus, the optimal DoF $\frac{4}{3}$ can be achieved. This result is in line with [5]. However, due to quantization error, the interference cannot be aligned perfectly so that the interference leakage to the signal space will bring DoF performance loss. In the following, we will investigate the impact of quantization error on the achieved DoF.

\section{B. DoF analysis}

To analyze the DoF performance, we first provide the following lemma.

Lemma 1: The equivalent MAT scheme described in the above subsection achieves the following sum DoF

$$
d=\frac{4}{3}-\frac{2}{3}\left(1+\lim _{P \rightarrow \infty} \frac{\mathbb{E}\left[\log _{2}\left(\sigma_{2}^{2}\right)\right]}{\log _{2}(P)}\right)^{+},
$$

where $\sigma_{2}$ is the second largest singular value of $\mathbf{I}_{D_{2}}$.

Proof: Define the interference matrix at $D_{2}$ as $\mathbf{I}_{D_{1}}$. Combining $\mathbf{y}_{D_{1}}$ and the Lemma $\mathbf{1}$ in [4], and considering the fact that $\mathbf{I}_{D_{2}}$ and $\mathbf{I}_{D_{1}}$ have the same distribution, we can derive (13).

Based on Lemma 1, if we can find the relation of $\sigma_{2}$ and the quantization errors occurred in both hops, i.e., $\sin \left(\theta_{f}\right)$ and $\sin \left(\theta_{g}\right)$, then using (6), we may relate the achieved sum DoF with quantization rate. To this end, one may try to derive the exact expression of $\sigma_{2}$, as in [4]. However, in the considered network, the interference matrix $\mathbf{I}_{D_{2}}$ contains the channel information regarding two hops. Directly deriving $\sigma_{2}$ regarding $\sin \left(\theta_{f}\right)$ and $\sin \left(\theta_{g}\right)$ is much more involved. Thus, to solve the problem, we first provide an upper-bound to $\mathbb{E}\left[\log _{2}\left(\sigma_{2}^{2}\right)\right]$ in the following lemma and then relate the upper bound to quantization errors.

Lemma 2: Consider quantization codebooks $\mathcal{A}_{R}=$ $\left\{\hat{\mathbf{r}}_{1}, \hat{\mathbf{r}}_{2}, \ldots, \hat{\mathbf{r}}_{2^{B} R}\right\}$ and $\mathcal{A}_{\mathcal{D}}=\left\{\hat{\mathbf{d}}_{1}, \hat{\mathbf{d}}_{2}, \ldots, \hat{\mathbf{d}}_{2^{B} D}\right\}$ at the relays and destinations respectively. $\mathbb{E}\left[\log _{2}\left(\sigma_{2}^{2}\right)\right]$ can be upperbounded as follows:

$$
\mathbb{E}\left[\log _{2}\left(\sigma_{2}^{2}\right)\right] \leq \log _{2}\left[1-\cos \left(\theta_{f}\right) \cos \left(\theta_{g}\right)\right],
$$

where $\cos \left(\theta_{f}\right)=\sqrt{1-\sin ^{2}\left(\theta_{f}\right)}=\max _{1 \leq k<l \leq 2^{B_{R}}}\left|\hat{\mathbf{r}}_{k}^{*} \hat{\mathbf{r}}_{l}\right|$, and $\cos \left(\theta_{g}\right)=\sqrt{1-\sin ^{2}\left(\theta_{g}\right)}=\max _{1 \leq k<l \leq 2^{B_{D}}}\left|\hat{\mathbf{d}}_{k}^{*} \hat{\mathbf{d}}_{l}\right|$. 
Proof: Please see Appendix A.

Using Lemma 1 and Lemma 2, now we can have the following theorem.

Theorem 2: For the 2-user 2-hop MISO broadcast network with global-range quantized delayed feedback, the achieved sum DoF by the equivalent MAT scheme can be lowerbounded by

$$
d \geq \frac{4}{3}-\frac{2}{3}\left(1+\lim _{P \rightarrow \infty} \frac{-\min \left\{B_{R}, B_{D}\right\}}{\log _{2}(P)}\right)^{+},
$$

If the quantization rates scale linearly with $P$ as $B_{R}=$ $\alpha_{1} \log _{2}(P)$ and $B_{D}=\alpha_{2} \log _{2}(P)$, we have

$$
d \geq \frac{4}{3}-\frac{2}{3}\left(1-\min \left\{\alpha_{1}, \alpha_{2}\right\}\right)^{+} .
$$

Proof: Please see Appendix B.

Clearly, the result provided in Theorem 2 implies that when $\min \left\{\alpha_{1}, \alpha_{2}\right\} \geq 1$, the optimal sum DoF $\frac{4}{3}$ can be achieved.

\section{ONE-HOP-RANGE QUANTIZED DELAYED FEEDBACK}

With global-range feedback, the considered network can be seen by the source as an equivalent MISO broadcast channel. Thus the equivalent MAT scheme is applicable. However, with one-hop feedback, the source has the channel knowledge regarding only the first hop. The above perspective no longer holds. Based on this point, we consider another IA-based twophase transmission scheme.

\section{A. Transmission scheme}

Phase one: Individual message broadcast. This phase takes 2 time slots and works the same as the first phase of the equivalent MAT scheme in Section IV-A. Therefore, at time slot $t(t \in\{1,2\})$, the received signals $y_{R_{i}}(t), y_{D_{i}}(t)$, and $\bar{y}_{D_{i}}(t)=y_{D_{i}}(t) /\left|\mathbf{g}_{i}(t)\right|, i \in\{1,2\}$, can again be calculated by (7)-(9). But different from the previous section, after the second time slot, only the quantized channel vectors $\hat{\mathbf{f}}_{i}(1)$ and $\hat{\mathbf{f}}_{i}(2)$ are known at the source. The knowledge of $\hat{\mathbf{g}}_{i}(1)$ and $\hat{\mathrm{g}}_{i}(2)$ is available only at the relays.

Phase two: Common equation delivery. In the third time, the source combines the messages $\mu_{1}, \nu_{1}, \mu_{2}, \nu_{2}$, and transmits

$$
\mathbf{x}(3)=\left[\hat{y}_{R_{2}}(1)+\hat{y}_{R_{1}}(2) 0\right]^{T},
$$

where $\hat{y}_{R_{i}}(t)$ represents the signal in (7) with the channel vector $\mathbf{f}_{i}(t)$ replaced by $\hat{\mathbf{f}}_{i}(t)$. The received signal at $R_{i}$ is

$$
y_{R_{i}}(3)=f_{i 1}(3)\left(\hat{y}_{R_{2}}(1)+\hat{y}_{R_{1}}(2)\right) .
$$

Since $f_{11}(3)$ is available at $R_{1}$, let $\check{y}_{R_{2}}(1)=\frac{y_{R_{1}}(3)}{f_{11}(3)}-\bar{y}_{R_{1}}(2)$ and $R_{1}$ would have a corrupted version of $y_{R_{2}}(1)$ :

$$
\check{y}_{R_{2}}(1)=\hat{y}_{R_{2}}(1)+\hat{y}_{R_{1}}(2)-\bar{y}_{R_{1}}(2)=\hat{y}_{R_{2}}(1)+\epsilon_{1},
$$

where $\epsilon_{1}=\hat{y}_{R_{1}}(2)-\bar{y}_{R_{1}}(2)$ represents an error leakage due to quantization. Thus, combing the quantized delayed CSI vector $\hat{\mathrm{g}}_{2}(1)$, a corrupted version of $y_{D_{2}}(1)$ can be constructed as

$$
\check{y}_{D_{2}}(1)=\left[\hat{g}_{21}(1) \hat{g}_{22}(1)\right]\left[\begin{array}{c}
\bar{y}_{R_{1}}(1) \\
\check{y}_{R_{2}}(1)
\end{array}\right] .
$$

Similarly, setting $\epsilon_{2}=\hat{y}_{R_{1}}(2)-\bar{y}_{R_{2}}(1), R_{2}$ can compute

$$
\check{y}_{R_{1}}(2)=\hat{y}_{R_{2}}(1)+\hat{y}_{R_{1}}(2)-\bar{y}_{R_{2}}(1)=\hat{y}_{R_{1}}(2)+\epsilon_{2},
$$

and construct a corrupted version of $y_{D_{1}}(2)$

$$
\check{y}_{D_{1}}(2)=\left[\hat{g}_{11}(2) \hat{g}_{12}(2)\right]\left[\begin{array}{l}
\check{y}_{R_{1}}(2) \\
\bar{y}_{R_{2}}(2)
\end{array}\right] .
$$

Set the transmission signals at relays as $x_{R_{1}}(3)=\check{y}_{D_{2}}(1)$ and $x_{R_{2}}(3)=\check{y}_{D_{1}}(2)$. The received signals at destinations are

$$
y_{D_{i}}(3)=g_{i 1}(3) \check{y}_{D_{2}}(1)+g_{i 2}(3) \check{y}_{D_{1}}(2), \quad i \in\{1,2\} \text {. }
$$

Then the received signals at $D_{1}$ during the three time slots can be expressed by a matrix format: $\mathbf{y}_{D_{1}}=$ $\left[\bar{y}_{D_{1}}(1) \bar{y}_{D_{1}}(2) \frac{y_{D_{1}}(3)}{g_{12}(3)}\right]^{T}$, i.e.

$$
\mathbf{y}_{D_{1}}=\mathbf{H}_{D_{1}}\left[\begin{array}{c}
\mu_{1} \\
\nu_{1}
\end{array}\right]+\mathbf{I}_{D_{2}}\left[\begin{array}{c}
\mu_{2} \\
\nu_{2}
\end{array}\right]+\left[\begin{array}{c}
0 \\
0 \\
\rho \epsilon_{1}
\end{array}\right],
$$

where the equivalent channel matrix $\mathbf{H}_{D_{1}}$ can be derived according (9) and (23) and can be shown to have rank 2 almost surely, the interference matrix $\mathbf{I}_{D_{2}}$ is (we omit the time index $t=2$ of each channel coefficient)

$$
\mathbf{I}_{D_{2}}=\left[\begin{array}{cc}
0 & 0 \\
\bar{g}_{11} \bar{f}_{11}+\bar{g}_{12} \bar{f}_{21} & \bar{g}_{11} \bar{f}_{12}+\bar{g}_{12} \bar{f}_{22} \\
\hat{g}_{11} \hat{f}_{11}+\hat{g}_{12} \bar{f}_{21} & \hat{g}_{11} \hat{f}_{12}+\hat{g}_{12} \bar{f}_{22}
\end{array}\right] .
$$

It must be noted that the $\mathbf{I}_{D_{2}}$ in (25) is slightly different from what we obtain in global-range feedback scenario. $\rho \epsilon_{1}$ represents the error leakage, and $\rho=\frac{g_{11}(3)}{g_{12}(3)} \hat{g}_{22}(1)$. Using (6), the power of the error leakage can be upper bounded by

$$
\left|\epsilon_{i}\right|^{2} \leq P\left(1-\cos \left(\theta_{f}\right)\right) \leq P \sin ^{2}\left(\theta_{f}\right), \forall i=1,2 .
$$

From (24), we can observe that if no quantization error occurs, the error leakage $\epsilon_{1}$ will vanish and $\mathbf{I}_{D_{2}}$ will have rank 1 almost surely. In addition, the signal space spanned by $\mathbf{H}_{D_{1}}$ has no overlapping with the interference space spanned by $\mathbf{I}_{D_{2}}$. By zero-forcing the interference, the DoF $\frac{2}{3}$ can be achieved. We can make the same conclusion for $D_{2}$ analogously. Hence, the optimal sum DoF $\frac{4}{3}$ is achieved by such an IA-based transmission scheme. However, if quantization error is not zero, interference cannot be completely nulled and the error leakage $\epsilon_{1}$ will be notable. How they affect the DoF performance will be studied in the following subsection.

\section{B. DoF analysis}

Equation (24) exhibits $\mathbf{y}_{D_{1}}$, the received signal vector at $D_{1}$. We can also follow the similar approach to have $\mathbf{y}_{D_{2}}$, with interference matrix $\mathbf{I}_{D_{1}}$. It is clear that $\mathbf{I}_{D_{1}}$ and $\mathbf{I}_{D_{2}}$ have the same distribution. Then we can obtain the following lemma.

Lemma 3: The sum DoF achieved by the above IA-based transmission scheme can be lower bounded as follows:

$$
d \geq \frac{4}{3}-\frac{2}{3}\left(1+\lim _{P \rightarrow \infty} \frac{\mathbb{E}\left[\log _{2}\left(\sigma_{2}^{2}\right)\right]+\epsilon}{\log _{2}(P)}\right)^{+},
$$

where $\sigma_{2}$ is the second largest singular value of $\mathbf{I}_{D_{2}}$, and $\epsilon=\log _{2}\left(1+\frac{P}{2^{B} R^{-1}}\right)$. 
Proof: Please see Appendix C.

We can observe that the DoF loss is induced by two factors: the interference leakage on the signal space causes the term $\frac{\mathbb{E}\left[\log _{2}\left(\sigma_{2}^{2}\right)\right]}{\log _{2}(P)}$ and the quantization error leakage in the first hop causes the term $\frac{\epsilon}{\log _{2}(P)}$. Therefore, to obtain the optimal sum DoF, we should guarantee both terms to be properly bounded.

To minimize quantization error leakage, the straightforward approach is to apply a sufficiently high quantization rate. For instance, let $B_{R}$ scale with $\log _{2}(P)$ as $B_{R}=\alpha_{1} \log _{2}(P)$. Then we have $\lim _{P \rightarrow \infty} \frac{\epsilon}{\log _{2}(P)}=\left(1-\alpha_{1}\right)^{+}$.

To bound the impact of the singular value $\sigma_{2}$, we adopt the similar approach in Lemma 2. Specifically, following the procedure described in Appendix A, we substitute (25) into (31) and end up with the following bound:

$$
\mathcal{F}\left(\mathbb{E}\left[\tilde{\mathbf{I}}_{D_{2}} \tilde{\mathbf{I}}_{D_{2}}^{*}\right]\right) \leq 1-\cos \left(\theta_{f}\right) \cos \left(\theta_{g}\right) .
$$

Thus we have the same upper bound on $\mathbb{E}\left[\log _{2}\left(\sigma_{2}^{2}\right)\right]$ :

$$
\mathbb{E}\left[\log _{2}\left(\sigma_{2}^{2}\right)\right] \leq \log _{2}\left[1-\cos \left(\theta_{f}\right) \cos \left(\theta_{g}\right)\right] .
$$

Now if we also let $B_{D}$ linear scale with $\log _{2}(P)$, we can have the following theorem.

Theorem 3: For the 2-user 2-hop MISO broadcast network with one-hop-range quantized delayed feedback, if the quantization rates at relays $B_{R}=\alpha_{1} \log _{2}(P)$ and at destinations $B_{D}=\alpha_{2} \log _{2}(P)$, the achieved sum DoF by the IA-based transmission scheme can be lower bounded as follows:

$$
d \geq \frac{4}{3}-\frac{2}{3}\left(\left(1-\min \left\{\alpha_{1}, \alpha_{2}\right\}\right)^{+}+\left(1-\alpha_{1}\right)^{+}\right) .
$$

Proof: The proof follows the approach in Appendix B.

Clearly, when $\alpha_{1} \geq 1$ then the lower bound of the achieved DoF with one-hop-range feedback is the same as that with global-range feedback shown in (16). Otherwise the lower bound we obtained in (30) would be smaller than that in (16). The quantization error leakage of the first hop may cause further performance degradation. Therefore, providing the first hop high quantization quality may be more crucial for obtaining high DoF performance in the one-hop-range feedback scenario. Furthermore, when the quantization rates of both hops are are sufficiently high such that $\min \left(\alpha_{1}, \alpha_{2}\right) \geq 1$, the optimal DoF $\frac{4}{3}$ can be achieved. This result is consistent with that in the global-range feedback scenario.

\section{Conclusions}

We have considered communication in a downlink multihop MISO network with quantized delayed CSIT: two singleantenna relays are deployed to establish transmission links from a two-antenna source to two single-antenna destinations. The source may decode the quantized delayed CSI feedback signals from both hops, or feedback can occur within only each hop. For each scenario, we consider a spectrally efficient transmission scheme and establish a lower bound of the achieved sum DoF. Our results have shown that when the quantization rate at every terminal is $\alpha \log _{2}(S N R)$, the optimal sum DoF can be achieved for $\alpha \geq 1$ in the considered network.

\section{APPENDIX A}

PROOF OF LEMMA 2

We define $\mathcal{F}(\mathbf{A})$ as the function which finds the smallest eigenvalue of matrix $\mathbf{A}$. Let $\tilde{\mathbf{I}}_{D_{2}}$ be a $2 \times 2$ matrix constituted by the last two rows of $\mathbf{I}_{D_{2}}$. Due to the concavity of $\log (\cdot)$ function and Jensen's inequality, we have

$$
\begin{aligned}
\mathbb{E}\left[\log _{2}\left(\sigma_{2}^{2}\right)\right] & \leq \log _{2}\left[\mathbb{E}\left(\sigma_{2}^{2}\right)\right] \\
& =\log _{2}\left\{\mathbb{E}\left(\mathcal{F}\left(\tilde{\mathbf{I}}_{D_{2}} \tilde{\mathbf{I}}_{D_{2}}^{*}\right)\right)\right\} \\
& \leq \log _{2}\left\{\mathcal{F}\left(\mathbb{E}\left(\tilde{\mathbf{I}}_{D_{2}} \tilde{\mathbf{I}}_{D_{2}}^{*}\right)\right)\right\}
\end{aligned}
$$

where (33) follows the fact that finding the smallest eigenvalue of a hermitian matrix is a concave function [10].

Because the channel coefficients follow i.i.d. Rayleigh distribution, after some mathematical manipulation the expectation of the matrix $\tilde{\mathbf{I}}_{D_{2}} \tilde{\mathbf{I}}_{D_{2}}^{*}$ can be expressed as:

$$
\begin{aligned}
& \mathbb{E}\left[\tilde{\mathbf{I}}_{D_{2}} \tilde{\mathbf{I}}_{D_{2}}^{*}\right] \\
= & {\left[\begin{array}{cc}
1 & \mathbb{E}\left(\bar{f}_{11}^{*} \hat{f}_{11} \overline{\mathbf{g}}_{1} \hat{\mathbf{g}}_{1}^{*}+\bar{f}_{12}^{*} \hat{f}_{12} \overline{\mathbf{g}}_{2} \hat{\mathbf{g}}_{2}^{*}\right) \\
\mathbb{E}\left(\bar{f}_{11} \hat{f}_{11}^{*} \overline{\mathbf{g}}_{1}^{*} \hat{\mathbf{g}}_{1}+\bar{f}_{12} \hat{f}_{12}^{*} \overline{\mathbf{g}}_{2}^{*} \hat{\mathbf{g}}_{2}\right) & 1
\end{array}\right] }
\end{aligned}
$$

Consider the worst case so that $\left|\overline{\mathbf{g}}_{i}^{*} \hat{\mathbf{g}}_{i}\right|=\cos \left(\theta_{g}\right)$ and $\left|\overline{\mathbf{f}}_{i}^{*} \hat{\mathbf{f}}_{i}\right|=$ $\cos \left(\theta_{f}\right)$. The eigenvalue of $\mathbb{E}\left[\tilde{\mathbf{I}}_{D_{2}} \tilde{\mathbf{I}}_{D_{2}}^{*}\right]$ can be computed as

$$
\mathcal{F}\left(\mathbb{E}\left[\tilde{\mathbf{I}}_{D_{2}} \tilde{\mathbf{I}}_{D_{2}}^{*}\right]\right)=1-\cos \left(\theta_{f}\right) \cos \left(\theta_{g}\right) .
$$

Substituting (34) into (33) completes the proof.

\section{APPENDIX B}

PROOF OF THEOREM 2

We define $\Delta d=\frac{2}{3}\left(1+\lim _{P \rightarrow \infty} \frac{\mathbb{E}\left[\log _{2}\left(\sigma_{2}^{2}\right)\right]}{\log _{2}(P)}\right)^{+}$. Lemma 2 leads to

$$
\Delta d \leq \frac{2}{3}\left(1+\lim _{P \rightarrow \infty} \frac{\mathbb{E}\left[\log _{2}\left(1-\cos \left(\theta_{f}\right) \cos \left(\theta_{g}\right)\right)\right]}{\log _{2}(P)}\right)^{+} .
$$

Considering the upper bound of quantization error in (6), we have $\sin ^{2}\left(\theta_{f}\right) \leq 2^{1-B_{R}}$ and $\sin ^{2}\left(\theta_{g}\right) \leq 2^{1-B_{D}}$. Hence, we have

$$
\begin{aligned}
& \lim _{P \rightarrow \infty} \frac{\mathbb{E}\left[\log _{2}\left(1-\cos \left(\theta_{f}\right) \cos \left(\theta_{g}\right)\right)\right]}{\log _{2}(P)} \\
\leq & \lim _{P \rightarrow \infty} \frac{\log _{2}\left(1-\sqrt{\left(1-2^{1-B_{R}}\right)\left(1-2^{1-B_{D}}\right)}\right)}{\log _{2}(P)} \\
\leq & \lim _{P \rightarrow \infty} \frac{1-\min \left\{B_{R}, B_{D}\right\}}{\log _{2}(P)} \\
= & \lim _{P \rightarrow \infty} \frac{-\min \left\{B_{R}, B_{D}\right\}}{\log _{2}(P)}
\end{aligned}
$$

Based on (39), we have

$$
\Delta d \leq \frac{2}{3}\left(1+\lim _{P \rightarrow \infty} \frac{-\min \left\{B_{R}, B_{D}\right\}}{\log _{2}(P)}\right)^{+} .
$$

Considering the case that $B_{R}=\alpha_{1} \log _{2}(P)$ and $B_{D}=$ $\alpha_{2} \log _{2}(P)$, we have

$$
\Delta d \leq \frac{2}{3}\left(1-\min \left\{\alpha_{1}, \alpha_{2}\right\}\right)^{+} .
$$

By substituting (41) into (13), we can finish the proof. 


\section{APPENDIX C \\ PROOF OF LEMMA 3}

We start from the received signals at destination $D_{1}$ as denoted in (24). Suppose $\mathbf{U}_{D_{1}}$ is a $2 \times 3$ zero-forcing matrix with rank 2 and $\mathbf{U}_{D_{1}} \mathbf{U}_{D_{1}}^{*}=\mathbf{I}$. We have the zero-forced signal at $D_{1}$ as follows:

$$
\mathbf{U}_{D_{1}} \mathbf{y}_{D_{1}}=\mathbf{U}_{D_{1}} \mathbf{H}_{D_{1}}\left[\begin{array}{c}
\mu_{1} \\
\nu_{1}
\end{array}\right]+\mathbf{U}_{D_{1}} \mathbf{I}_{D_{2}}\left[\begin{array}{c}
\mu_{2} \\
\nu_{2}
\end{array}\right]+\mathbf{U}_{D_{1}} \mathbf{e}_{1},
$$

where $\mathbf{e}_{1}=\left[\begin{array}{lll}0 & 0 & \rho \epsilon_{1}\end{array}\right]^{T}$. Then the average throughput of $D_{1}$ can be written as:

$\mathcal{R}_{1}=\frac{1}{3} \mathbb{E}\left[\log _{2}\left|\frac{\frac{P}{2} \mathbf{U}_{D_{1}} \mathbf{H}_{D_{1}} \mathbf{H}_{D_{1}}^{*} \mathbf{U}_{D_{1}}^{*}+\mathbf{M}+\mathbf{U}_{D_{1}} \mathbf{e}_{1} \mathbf{e}_{1}^{*} \mathbf{U}_{D_{1}}^{*}}{\mathbf{M}+\mathbf{U}_{D_{1}} \mathbf{e}_{1} \mathbf{e}_{1}^{*} \mathbf{U}_{D_{1}}^{*}}\right|\right]$

with

$$
\mathbf{M}=\mathbf{I}+\frac{P}{2} \mathbf{U}_{D_{1}} \mathbf{I}_{D_{2}} \mathbf{I}_{D_{2}}^{*} \mathbf{U}_{D_{1}}^{*},
$$

where $\mathbf{I}$ is a $2 \times 2$ identity matrix. Since $\mathbf{e}_{1}$ is the quantization error leakage at the first hop, it can not be obtained by the destination $D_{1} . D_{1}$ can only choose $\mathbf{U}_{D_{1}}$ to zero-force as much interference as possible. Define the singular value decomposition such that $\mathbf{I}_{D_{2}}=\mathbf{U} \boldsymbol{\Sigma} \mathbf{V}^{*}$. We choose $\mathbf{U}_{D_{1}}$ to be the last two rows of $\mathbf{U}^{*}$ such that the minimum value of $\operatorname{det}(\mathbf{M})$ is achieved as follows:

$$
\operatorname{det}(\mathbf{M})=1+\frac{P}{2} \sigma_{2}^{2},
$$

with $\mathbf{M}=\operatorname{diag}\left\{1+\frac{P}{2} \sigma_{2}^{2}, 1\right\}$. By representing $\mathbf{U}_{D_{1}}=$ $\left[\begin{array}{lll}\mathbf{u}_{1} & \mathbf{u}_{2} & \mathbf{u}_{3}\end{array}\right]$, we have:

$$
\operatorname{det}\left(\mathbf{M}+\mathbf{U}_{D_{1}} \mathbf{e}_{1} \mathbf{e}_{1}^{*} \mathbf{U}_{D_{1}}^{*}\right)=\operatorname{det}\left(\mathbf{M}+\left|\rho \epsilon_{1}\right|^{2} \mathbf{u}_{3} \mathbf{u}_{3}^{*}\right) .
$$

By Sylvester's determinant theorem [11], we have

$$
\begin{aligned}
\operatorname{det}\left(\mathbf{M}+\left|\rho \epsilon_{1}\right|^{2} \mathbf{u}_{3} \mathbf{u}_{3}^{*}\right) & =\operatorname{det}(\mathbf{M})\left(1+\left|\rho \epsilon_{1}\right|^{2} \mathbf{u}_{3}^{*} \mathbf{M}^{-1} \mathbf{u}_{3}\right) \\
& \stackrel{\text { (a) }}{\leq} \operatorname{det}(\mathbf{M})\left(1+\left|\rho \epsilon_{1}\right|^{2}\right) \\
& \stackrel{\text { (b) }}{\leq} \operatorname{det}(\mathbf{M})\left(1+|\rho|^{2} P \sin ^{2}\left(\theta_{f}\right)\right) \\
& \stackrel{\text { (c) }}{\leq} \operatorname{det}(\mathbf{M})\left(1+|\rho|^{2} \frac{P}{2^{B_{f}-1}}\right)
\end{aligned}
$$

where (a) follows from $\mathbf{u}_{3}^{*} \mathbf{M}^{-1} \mathbf{u}_{3}$ is always less or equal to the maximum eigenvalue of matrix $\mathbf{M}^{-1}$ for any vector $\mathbf{u}_{3}^{H} \mathbf{u}_{3} \leq 1$ (Rayleigh-Ritz theorem in [12]); (b) follows from (26); and (c) follows from the upper-bound on the quantization error (6).

Then, we have the achieved DoF at $D_{1}$ represented as

$$
\begin{aligned}
d_{1} & =\lim _{P \rightarrow \infty} \frac{\mathcal{R}_{1}(P)}{\log _{2}(P)} \\
& \stackrel{\text { (a) }}{=} \frac{2}{3}-\frac{1}{3} \lim _{P \rightarrow \infty} \frac{\mathbb{E}\left[\log _{2}\left|\mathbf{M}+\mathbf{U}_{D_{1}} \mathbf{e}_{1} \mathbf{e}_{1}^{*} \mathbf{U}_{D_{1}}^{*}\right|\right]}{\log _{2}(P)} \\
& \stackrel{\text { (b) }}{\geq} \frac{2}{3}-\frac{1}{3} \lim _{P \rightarrow \infty} \frac{\mathbb{E}\left[\log _{2}\left[\left(1+\frac{P}{2} \sigma_{2}^{2}\right)\left(1+\frac{P}{2^{B_{f}-1}}\right)\right]\right]}{\log _{2}(P)} \\
& =\frac{2}{3}-\frac{1}{3}\left(1+\lim _{P \rightarrow \infty} \frac{\mathbb{E}\left[\log _{2}\left(\sigma_{2}^{2}\right)\right]+\epsilon}{\log _{2}(P)}\right)^{+},
\end{aligned}
$$

with $\epsilon=\log _{2}\left(1+\frac{P}{2^{B_{R-1}}}\right)$. In the above equations, (a) follows from the signal matrix $\mathbf{H}_{D_{1}}$ has rank 2 almost surely and the signal power dominates the interference power as $P \rightarrow \infty$; (b) follows by substituting (46) and $\mathbb{E}\left[|\rho|^{2}\right]=1$.

The achieved DoF for destination $D_{2}$ can be analyzed in the similar derivation to finish the proof of this lemma.

\section{REFERENCES}

[1] N. Jindal, "MIMO broadcast channels with finite-rate feedback," IEEE Trans. Inf. Theory, vol. 52, pp. 5045-5060, Nov. 2006.

[2] J. Zhang, M. Kountouris, J. G. Andrews, and R. W. Heath, "Multi-mode transmission for the MIMO broadcast channel with imperfect channel state information," IEEE Trans. Commun., vol. 59, pp. 803-814, Mar. 2011.

[3] M. A. Maddah-Ali and D. Tse, "Completely stale transmitter channel state information is still very useful," in 2010 Allerton Conference.

[4] J. Xu, J. G. Andrews, and S. A. Jafar, "MISO broadcast channels with delayed finite-rate feedback: predict or observe?" IEEE Trans. Wireless Commun., vol. 11, pp. 1456-1467, Apr. 2012.

[5] Z. Wang, M. Xiao, C. Wang, and M. Skoglund, "Degrees of freedom of relay-aided MIMO broadcast network with delayed CSIT," IEEE Wireless Commun. Lett., Oct. 2012, submitted. [Online]. Available: http://arxiv.org/abs/1210.3563

[6] J. Thukral and H. Bolskei, "Interference alignment with limited feedback," in Proc. IEEE Int. Symp. Information Theory, 2009.

[7] D. J. Love, R. W. Heath, and T. Strohmer, "Grassmannian beamforming for multiple-input multiple-output wireless systems," IEEE Trans. Inf. Theory, vol. 49, pp. 2735-2747, Oct. 2003.

[8] J. H. Conway, R. H. Hardin, and N. J. A. Sloane, "Packing lines, planes, etc.: Packings in Grassmannian spaces," Exper. Math., vol. 5, pp. 139159, Apr. 1996.

[9] A. Barg and D. Y. Nogin, "Bounds on packings of spheres in the Grassmannian manifold," IEEE Trans. Inf. Theory, vol. 48, pp. 24502454, Sep. 2002.

[10] T. Tao, Topics in Random Matrix Teory. American Mathematical Soc., 2012.

[11] D. A. Harville, Matrix Algebra from a Statistician's Perspective. Springer, 2008.

[12] R. A. Horn and C. R. Johnson, Matrix Analysis. Cambridge University Press, 1990. 